\title{
Outcomes of laparoscopic nissen fundoplications in children younger than 2-years: single institution experience
}

\author{
Armando Rosales $^{1} \cdot$ Jill Whitehouse $^{2} \cdot$ Carrie Laituri $^{2} \cdot$ Glenda Herbello $^{2} \cdot$ Julie Long $^{2}$ (I)
}

Accepted: 14 May 2018 / Published online: 28 May 2018

c) Springer-Verlag GmbH Germany, part of Springer Nature 2018

\begin{abstract}
Background Variation exists in the diagnostic testing for gastroesophageal reflux (GER) in infants and in the application of surgical therapy. There has been an increase in antireflux surgery (ARS) since the development of laparoscopy but the outcomes in high-risk infants is unclear. This study examines the results of laparoscopic fundoplication in infants less than 2 years.

Methods The results of infants less than 2 years undergoing laparoscopic Nissen fundoplication (Lap-F) from 2012 to 2015 were retrospectively reviewed and outcomes were followed until 2017.

Results There were 106 patients, median gestational age 32.50 weeks $\pm 6.35 \mathrm{SD}$ and non-corrected age at operation 23.0 weeks $\pm 19.0 \mathrm{SD}$, mean weight of $4.81 \mathrm{~kg} \pm 2.10 \mathrm{SD}$. One of the most common reasons for surgical consultation was improvement in respiratory status after insertion of nasoduodenal feeding tube. Of the Lap-F, 100 were with gastrostomy tube (GT). There were no conversions to open or intraoperative complications. The complication rate was $4.71 \%$, and the reoperation rate was $5.66 \%$, one fundoplication revision and the others gastrostomy revisions. The median time for feeds and to reach goal were $1(1-14)$ and 4 (2-279) days, respectively. The 30-day mortality was $0.9 \%$ and long-term it was $4.71 \%$. The long-term mortality was related to the underlying medical problems. The median follow-up was 113 (3-286) weeks. One patient required revision of the fundoplication and none required esophageal dilatation during the follow-up period.

Conclusion Fundoplication is effective for relief of symptoms of GER in children younger than 2 years. The procedure has a low morbidity and mortality in this population.
\end{abstract}

Keywords Laparoscopic $\cdot$ Nissen $\cdot$ Fundoplication $\cdot$ Gastroesophageal reflux

Project presented: World Federation of Associations of Pediatric Surgeons (WOFAPS) 2016 in Washington, DC.

Julie Long

Julielong@earthlink.net

Armando Rosales

rosalesarmando@gmail.com

Jill Whitehouse

jwhitehouse@mhs.net

Carrie Laituri

claituri@mhs.net

Glenda Herbello

gherbello@mhs.net

1 Department of Surgery, Cleveland Clinic Florida, Weston, USA

2 Department of Pediatric Surgery, Joe DiMaggio Children's Hospital, 1150 N 35th Ave, Suite 555, Hollywood, FL 33021, USA

\section{Introduction}

Gastroesophageal reflux (GER) is considered normal in infancy. It can be difficult and subjective to determine when to diagnosis gastroesophageal reflux disease (GERD) in an infant. When GER impacts the child's health, the diagnosis of GERD is made. In an infant with multiple medical problems it is particularly challenging to determine if GER is exacerbating underlying medical conditions. The underlying medical conditions and the treatment of these problems can also exacerbate the GER. The threshold for medical treatment of GERD varies between individual providers and institutions. There is greater institutional variation in surgical intervention for GERD. Diagnostic tests for GER are less accurate in infants and fail to distinguish between GER and GERD [1]. Besides regional variation in practice, the difficulty in accurately diagnosing reflux in infants is considerable. $\mathrm{PH}$ probe is considered the "gold standard" for 
detecting reflux but it does not indicate whether the infant has GERD and the decreased acid production in infants may decrease the usefulness of the test in sick infants $[2$, 3]. Upper gastrointestinal series is not considered sensitive enough for detecting reflux. It is considered useful to confirm anatomy and in $4 \%$ of cases can diagnose conditions that may influence management $[4,5]$. Query of the Pediatric Health Information System database revealed a wide variation in the diagnosis of GERD, testing performed and use of antireflux surgery (ARS) [6]. For some infants, the diagnosis of GERD is made based on clinical symptoms whereas other institutions seem to follow a practice of requiring confirmatory testing. In the most compromised infants requiring mechanical ventilation or with cardiac conditions it may be impossible to perform diagnostic testing to confirm GER.

Some pediatric hospitals use nasal or gastroduodenal feeding tubes for the management of infants with feeding problems and GERD, however there are significant issues with maintenance of these tubes and a $3 \%$ perforation rate [7]. A retrospective study utilized gastrojejunal feeding tubes in neurologically impaired children and found that $8.3 \%$ failed feeding tubes and required fundoplication [8]. No randomized trials have been done to evaluate if relief of vomiting, time in the hospital, complications or cost are better with transpyloric tubes or ARS. Just as indications for testing or diagnosing GERD vary, the threshold for recommending surgical treatment also varies widely between hospitals $[9,10]$. Perceived decreased morbidity of laparoscopic fundoplication may result in increased surgical intervention for GERD [10]. Certainly with most advanced laparoscopic procedures there is a significant learning curve and this can be a barrier to application of the procedure in high-risk infants.

Advances in neonatology and other pediatric specialties have improved the survival of medically complex children who often have feeding disorders, GER, GERD, pulmonary problems or growth failure. Optimal treatment would decrease symptoms believed to be caused by GERD, have a low risk of complications and be easily managed in an outpatient setting when the infant transitions to home. The aim of this study was to evaluate indications for laparoscopic fundoplication, the safety of the procedure and outcomes after hospital discharge in a group of medically complex infants under 2 years of age.

\section{Methods}

After Institutional Review Board (IRB) approval, data were collected retrospectively on all children younger than 2 years of age who underwent a laparoscopic fundoplication (Lap-F) with or without a gastrostomy tube (GT) from January 2012 through December 2015. No infant during that interval was excluded. Demographics, comorbidities, medical treatment and preoperative testing data were collected. The surgical team's indication for surgery was recorded. Also reviewed were complications, time to start feedings and reach goal, hospital length of stay and outpatient follow-up.

Infants with complex cardiac conditions received anesthesia by a pediatric cardiac anesthesiologist, whereas the other patients were anesthetized by a pediatric anesthesiologist. A standard procedure was followed for the surgery. Infants were placed in a frog leg position at the end of the operating table. For infants under $5 \mathrm{~kg}$, an 18 French orogastric tube was placed to decompress the stomach and left in place for the wrap. Larger children had this tube exchanged for an appropriately sized bougie for the wrap. Two 3-mm ports were place, one at the liver edge and one in the right lower quadrant. A 5-mm umbilical port was place and a 5-mm $30^{\circ}$ scope was used. At the site of the gastrostomy, a 5-mm port was placed. Initially another 5-mm trocar was placed in the left lower quadrant, but this was found to be unnecessary and was only used if the patient already had a previous gastrostomy. Carbon dioxide was insufflated to a pressure between 8 and $10 \mathrm{mmHg}$. A $360^{\circ}$ wrap was done in all cases except one. The esophagus was mobilized if there was a hiatal hernia. Short gastric vessels were divided in all cases. The hiatus was closed with one or two sutures if there was space noted after the esophageal mobilization. All infants received gastrostomy tubes unless they already had one in place.

\section{Results}

\section{Demographics and co-morbidities}

One hundred and six patients were included, 59 male (55\%) and 47 females (45\%). The median gestational age at birth was 32.50 weeks $\pm 6.35 \mathrm{SD}$ and the non-corrected age at operation was 23.0 weeks \pm 19.0 SD. Mean weight at surgery was $4.81 \pm 2.10 \mathrm{~kg}$. Sixty-three $(59.4 \%)$ of the infants were premature. Forty-eight patients were in the neonatal intensive care unit (NICU), 36 from the pediatric intensive care unit (PICU), seven from the intermediate care unit (IMCU) and one patient from the rehabilitation unit. Only 14 (13\%) were admitted as outpatients and referred by the pediatric gastroenterologists. Seventy-one (67\%) infants were hospitalized in an intensive care unit from birth until the time of operation.

Table 1 shows the demographics and comorbidities of the study sample. All infants had at least one medical problem in addition to their GERD. Medical problems included developmental delay $(63,59.4 \%)$, seizures $(16,15.1 \%)$, hydrocephalus $(13,12.3 \%), 5(4.7 \%)$ with shunt and $8(7.55 \%)$ without, chronic lung disease $(82,77.4 \%)$ with 28 (26.4\%) 
Table 1 Patient's demographics

\begin{tabular}{ll}
\hline Gender & \\
Male & $59(55)$ \\
Female & $47(45)$ \\
Median gestational age (weeks) & $32+6.3$ \\
Weight (kg) & $4.81 \pm 2$ \\
Noncorrected age at operation (weeks) & $23.0+19.0$ \\
Comorbidities & \\
Developmental delay & $63(59.4)$ \\
Hydrocephalus & $13(12.3)$ \\
$\quad$ Shunted & $5(4.7)$ \\
Pneumopathy & $82(77.4)$ \\
$\quad$ On ventilator & $28(26.4)$ \\
Cardiopathy & $59(55.6)$ \\
$\quad$ Cardiac surgery & $34(32.1)$ \\
Intraventricular hemorrhage & $24(22.6)$ \\
Seizures & $16(15.1)$ \\
Neuromuscular disease & $17(16)$ \\
Necrotizing enterocolitis & $4(3.7)$ \\
ASA score & \\
I & $1(1)$ \\
II & $5(4)$ \\
IV & $79(75)$ \\
\hline
\end{tabular}

Results represented in $N(\%)$, mean \pm standard deviation (SD) or median (range)

on ventilator support and 54 (51\%) with non-invasive support, congenital heart disease, 59, (55.6\%), 34 (32.1\%) postsurgical repair; intraventricular hemorrhage $(24,22.6 \%)$; necrotizing enterocolitis $(4,3.77 \%)$ and neuromuscular disease $(17,16.0 \%)$. Some infants had multiple medical problems. Sixteen (15.1\%) had a history of previous abdominal operations.

\section{Preoperative data}

Eight-five patients (80\%) were on medical therapy, of these, $60(75 \%)$ received a proton pump inhibitor and $25(25 \%)$ a $\mathrm{H} 2$ blocker. Conservative management was tried which included alteration in feeding volumes and interval, positional therapy, thickened feeds and transpyloric feeding tubes when nasogastric (NG) feedings were not tolerated. Diagnostic testing was not uniform because of the young age, size and medical status of the infants. PH probes, impedance probes, contrast studies including upper gastrointestinal series (UGI) and swallow studies were used at the discretion of the referring physician and occasionally requested by the consulting surgeon. Because of concerns about the accuracy of the $\mathrm{pH}$ probe in neonates and ventilated infants, it was rarely used. Fourteen (13.2\%) patients with GERD had $\mathrm{pH}$ probes. UGI was done in $94(88.7 \%)$ patients. Sixty-eight
Table 2 Preoperative work-up and positive results

\begin{tabular}{lcc}
\hline & Total (\%) & Positive (\%) \\
\hline Standard indication & & \\
UGI & $94(88.7)$ & $57(60.6)$ \\
pH probe & $14(13.2)$ & $9(69.2)$ \\
Esophageal biopsy & $6(5.7)$ & $6(100)$ \\
GERD in spite of medical treatment & $106(100)$ & $100(93.4)$ \\
Relative indication & & \\
ND improvement & $68(64.2)$ & $66(97.1)$ \\
Airway & $41(38.7)$ & $41(100)$ \\
Aspiration & $67(63.2)$ & $67(100)$ \\
Stricture & $4(3.8)$ & $4(100)$ \\
Consultant/parent request & $90(84.9)$ & $90(100)$ \\
\hline
\end{tabular}

Reported as $N(\%)$

(64.2\%) patients had transpyloric feeding tubes inserted for exacerbation of pulmonary problems thought to be feeding related and/or failure to gain weight with continuous gastric feeds associated with vomiting. Sixty-six $(97.1 \%)$ had clinical improvement with transpyloric tubes. Table 2.

Standard indications for surgery were a positive UGI, $\mathrm{pH}$ probe, esophageal biopsy and clinical reflux in spite of medical therapy. Relative indications for surgery were improvement in reflux symptoms with ND feedings, inability to protect the airway, suspicion for aspiration, a consultant or parent who requested surgery or an esophageal stricture. One hundred two infants had at least one standard indication for surgery and all patients had one or more relative indications for surgery.

\section{Operative data}

Six patients had Lap-F because of worsening reflux after gastrostomy only and 100 patients had Lap-F with gastrostomy performed concurrently. Operative time was $116.26+44.99 \mathrm{~min}$ (84 patients). Long gastrostomy tubes were used in 8 infants and 98 had button gastrostomy tubes placed. There were no conversions to open procedures and no intraoperative complications. Three patients required blood transfusion. A pediatric anesthesiologist assigned American Society for Anesthesiology score (ASA); as shown in Table 1 most patients were ASA $3(79,75 \%)$ or 4 $(21,20 \%)$.

\section{Postoperative data}

Feeds were started on postoperative day one based on the infants' condition; mean $1.54 \pm 1.26$ days. The mean time to reach goal feeds was $4.62 \pm 4.11$ days. Complications were identified in five patients (6\%), including leakage from the gastrostomy (3), pneumothorax (1) and wound infection 
(1). Time to reach goal feeds and hospitalization after surgery varied depending on the underlying medical condition. Postoperatively the median hospital stay was 14 days (2-70) and intensive care unit 52 days (0-440). For all the patients the median hospital stay was 56 days (2-284). The median hospital stay after surgery was 14 days (2-70). There were $6(5.6 \%)$ readmissions, including 3 for viral illnesses, 1 dislodged GT, and the 1 umbilical wound infection mentioned above.

There were five deaths total. There was one operative mortality defined by death within 30 days of operation. This infant died of multisystem organ failure. Four infants died during the follow-up period. One was found unresponsive at home 8 months after surgery and three from respiratory failure while still in the hospital (one of these children had been transferred to hospice).

\section{Follow-up}

After discharge from the hospital infants were followed by their surgeon and medical specialists. Mean follow up was $107.6 \pm 80.8$ weeks. Resolution of symptoms occurred in 93 $(87.7 \%)$ of infants. The gastrostomy was kept for a mean of 20 months in those patients who had had their tubes removed whereas the remainder was still using their tubes during the follow-up period. No infants required operation for bowel obstruction or wrap break down. One infant required revision of his fundoplication for GERD, the only infant who had a $180^{\circ}$ wrap. No infant required esophageal dilatation for a tight wrap. Recurrence of emesis occurred in eight patients that were managed non-operatively. Table 3 .

\section{Discussion}

GER is a very common condition in infancy. The classic teaching is infants without other medical conditions outgrow reflux by 8-10 months of age. GERD, however, frequently requires medical and/or surgical treatment in infants with other medical conditions. Some have stated that the perceived decrease morbidity from laparoscopic fundoplication has led to increase diagnosis of GERD and surgical treatment [11]. Paralleling new surgical techniques are advances in neonatal and pediatric intensive care that have improved survival for serious conditions and increased the need for supportive care.

In our institution, placement of a transpyloric feeding tube was initially used as a method for safely administering enteral feeds to infants with frequent vomiting or in intubated infants where silent reflux was thought to contribute to deterioration in pulmonary function. Improvement with transpyloric tubes prompted surgical consultation in many infants, especially those requiring mechanical ventilation.
Table 3 Postoperative results

\begin{tabular}{ll}
\hline Tube feeds & \\
Days to start & $1.54 \pm 1.26$ \\
Days to reach goal & $4.62 \pm 4.11$ \\
Hospitalization (days) & \\
Total hospitalization & $56(2-484)$ \\
Intensive care unit & $52(2-284)$ \\
Postoperative hospitalization & $14(2-70)$ \\
Complications & $5(6)$ \\
Leak & $3(60)$ \\
Pneumothorax & $1(10)$ \\
Wound infection & $1(10)$ \\
Readmission & $6(5.6)$ \\
Viral illness & $3(2.8)$ \\
Dislodged tube & $2(1.8)$ \\
Umbilical wound infection & $1(1)$ \\
Revision fundoplication & $1(1)$ \\
Reoperation initial hospitalization & $5(4.7)$ \\
G-tube revision & $3(2.8)$ \\
G-tube dislodged & $2(1.8)$ \\
\hline
\end{tabular}

Reported as $N(\%)$, mean \pm standard deviation (SD) or median (range)

The primary indications for LAP-N and GT were a positive $\mathrm{pH}$ probe or UGI, esophagitis and clinical reflux despite medical treatment. Relative indications for surgical intervention were a trial of ND feeds with improvement in GER symptoms, an infant who could not protect the airway or was suspected to have aspiration, a consultant or parent who requested fundoplication or an esophageal stricture. Some of the infants were on ventilator support for months before the decision was made to consider surgery based on improvement with transpyloric feeding. These were also the infants who had the longest time to achieve full feeds and discharge from the hospital. Those infants who had transpyloric tubes were refractory to medical therapy, had ongoing pulmonary problems and were managed by multiple intensivists over time. While treatment with transpyloric tubes was considered a relative indication for surgery, it was also used as a method to determine if reflux was contributing to the infants' problems. Increasingly over the time of this study, improvement with transpyloric feeding became an indication for surgery especially in infants who were not candidates for other diagnostic tests. Many of our infants had primary or secondary upper airway disease and/or vocal fold pathology making any reflux episode more dangerous. The association of apnea and bradycardia with GERD remains disputed. Blinman suggests that while GERD may not be causing the apnea, it may be causing more frequent relaxation of the lower esophageal sphincter [2]. Fundoplication and gastrostomy has been shown to be an effective treatment for infants with chronic airway disease and GERD $[12,13]$. In a large 
series of children with asthma, $80 \%$ were able to wean off steroids following Lap-F [14]. Therefore, this study suggests that improvement in respiratory symptoms and tolerance of feeds with transpyloric tubes may be an acceptable relative indication for surgical treatment of GERD.

In our hospital, prior to performing Lap-F, anecdotally we found the postoperative ileus, increased need for respiratory support and long term issues with bowel obstruction were a significant source of morbidity following fundoplication. Adhesive bowel obstruction occurs in $8 \%$ of children treated with open fundoplication versus none with Lap-F [15]. Our positive results with Lap-F increased requests for Lap-F from the intensivist, gastroenterologists and parents. Over a 20 year period, Dreissen et al. reported increased use of Lap-F to treat GERD in what was believed to be an otherwise stable surgical population supporting the concept of a change in referral attitudes [11].

Early reports of Lap-F in children suggested some benefit in terms of decreased respiratory symptoms but noted a higher need for reoperation compared to open fundoplication [16]. Shah studied Lap-F in infants weighing less than $5 \mathrm{~kg}$ and reported a 7\% conversion to open repair [17]. In a nonrandomized study evaluating infants less than 12 weeks old who underwent fundoplication, early results with either open or laparoscopic approaches were similar, suggesting the feasibility of the laparoscopic approach [18]. The possibility of performing Lap-F in children with previous abdominal operations was shown in a series with 45 children, none of whom required conversion to open fundoplication [19]. Citing no randomized studies evaluating open versus Lap-F, the American Pediatric Surgical Association (APSA) committee on new technology concluded that Lap-N is an acceptable treatment for medically refractory reflux but noted a possibly higher wrap failure rate and a significant learning curve [20]. A randomized trial comparing laparoscopic versus open fundoplication showed no difference in outcomes but higher operating costs in the laparoscopic group. They had a $23 \%$ conversion rate in the laparoscopic group, a higher percentage of prior abdominal surgery in the open group and did not present information about medical comorbidities [21]. Shariff et al. reported an $18 \%$ need for redo fundoplication and a $20 \%$ late mortality [22]. Rothenberg reported a $4.5 \%$ reoperation rate and noted this was highest in infants less than 6 months [23]. Laje et al. reported a $2.6 \%$ redo fundoplication rate in their series of children less than 2 years [24]. Similarly, our lack of recurrence or need for redo fundoplication and low complication rate supports this recent study and we believe it is safe to perform in medically complex children less than 2 years old.

This paper has several limitations. It is a retrospective review because our favorable experience with laparoscopic treatment of GERD in infants would make a randomized study of open versus Lap-F unacceptable to our referring physicians, patients and surgeons. In addition the current diagnostic testing for GERD and indications for surgery are evolving to address the needs of an expanding population of medically fragile infants but have not been studied in a randomized trial. In our community infants are not sent home with transpyloric feeding tubes unless they are in hospice, the families have refused surgical intervention or the infants are deemed unacceptable risks for general anesthesia so we have not compared this treatment to Lap-F. Finially, our series included different surgeons and numerous medical specialists all of whom contributed to the various choices in diagnostic studies.

\section{Conclusions}

Lap-F can be safely performed in a high risk group of infants less than 2 years of age. Improvements in respiratory status in infants treated with a transpyloric feeding tube for several weeks should be considered strong evidence that reflux is contributing to the pulmonary problems. This finding more reliably indicates those infants who will benefit from Lap-F than the usual testing done to check for reflux. We believe improvement in respiratory status with transpyloric feeds should be a standard indication for surgical treatment in infants who cannot undergo other testing. Based on our study and other reports of good outcomes with this operation, we believe that laparoscopic fundoplication is now the preferable way to treat medically refractory reflux in infants. A high risk group of infants with prematurity, chronic lung disease, cardiac problems, GERD and feeding problems can be treated with Lap-F and GT. Just as laparoscopic cholecystectomy has virtually replaced open cholecystectomy because of decreased pain and shorter recovery, laparoscopic fundoplication is replacing open fundoplication in high-risk infants with medical comorbidities and can be done safely in infants less than 2 years of age.

\section{Compliance with ethical standards}

Conflict of interest None of the authors report a conflict of interest.

Ethical approval This project was approved by the Institutional Review Board of Joe DiMaggio Children's Hospital.

Informed consent Informed consent was waived by the committee the study was retrospective and posed no risk to the participants. The study complied with the 1964 Helsinki declaration and its later amendments.

\section{References}

1. van der Pol RJ, Smits MJ, Venmans L, Boluyt N, Benninga MA, Tabbers MM (2013) Diagnostic accuracy of tests in pediatric 
gastroesophageal reflux disease. J Pediatr 162(5):983-987.e981984. https://doi.org/10.1016/j.jpeds.2012.10.041

2. Blinman T (2015) Gastroesophageal reflux and the neonatal airway. In: Lioy J, Sobol S (eds) Disorders of the neonatal airway. Springer, New York, pp 281-294. https://doi. org/10.1007/978-1-4939-1610-8_26

3. Lopez-Alonso M, Moya MJ, Cabo JA, Ribas J, del Carmen Macias M, Silny J, Sifrim D (2006) Twenty-four-hour esophageal impedance-pH monitoring in healthy preterm neonates: rate and characteristics of acid, weakly acidic, and weakly alkaline gastroesophageal reflux. Pediatrics 118(2):e299-308. https://doi. org/10.1542/peds.2005-3140

4. Cuenca AG, Reddy SV, Dickie B, Kays DW, Islam S (2011) The usefulness of the upper gastrointestinal series in the pediatric patient before anti-reflux procedure or gastrostomy tube placement. J Surg Res 170(2):247-252. https://doi.org/10.1016/j. jss.2011.03.007

5. Valusek PA, St Peter SD, Keckler SJ, Laituri CA, Snyder CL, Ostlie DJ, Holcomb GW III (2010) Does an upper gastrointestinal study change operative management for gastroesophageal reflux? J Pediatr Surg 45(6):1169-1172. https://doi.org/10.1016/j.jpeds urg.2010.02.083

6. Short HL, Braykov NP, Bost JE, Raval MV (2017) Variation in preoperative testing and antireflux surgery in infants. Pediatrics. https://doi.org/10.1542/peds.2017-0536

7. Campwala I, Perrone E, Yanni G, Shah M, Gollin G (2015) Complications of gastrojejunal feeding tubes in children. J Surg Res 199(1):67-71. https://doi.org/10.1016/j.jss.2015.06.058

8. Wales PW, Diamond IR, Dutta S, Muraca S, Chait P, Connolly B, Langer JC (2002) Fundoplication and gastrostomy versus imageguided gastrojejunal tube for enteral feeding in neurologically impaired children with gastroesophageal reflux. J Pediatr Surg 37(3):407-412

9. Barnhart DC (2016) Gastroesophageal reflux disease in children. Semin Pediatr Surg 25(4):212-218. https://doi.org/10.1053/j. sempedsurg.2016.05.009

10. Hassall E (2005) Decisions in diagnosing and managing chronic gastroesophageal reflux disease in children. J Pediatr 146(3 Suppl):S3-12. https://doi.org/10.1016/j.jpeds.2004.11.034

11. Driessen C, Verhoeven BH, Ten WE, Van Heurn LW (2010) Does laparoscopy lower the threshold for the surgical treatment of gastroesophageal reflux disease in children? J Pediatr Gastroenterol Nutr 51(5):599-602. https://doi.org/10.1097/MPG.0b013e3181 ddc014

12. Frongia G, Ahrens P, Capobianco I, Kossler-Ebs J, Stroh T, Fritsche R, Lettgen B, Kessler M, Mehrabi A, Gunther P, HollandCunz S (2015) Long-term effects of fundoplication in children with chronic airway diseases. J Pediatr Surg 50(1):206-210. https ://doi.org/10.1016/j.jpedsurg.2014.09.079

13. Macharia EW, Eaton S, de Coppi P, Curry J, Drake D, Cross K, Kiely E, Pierro A (2012) Fundoplication in ventilator-dependent infants with gastro-oesophageal reflux. Eur J Pediatr Surg 22(1):91-96. https://doi.org/10.1055/s-0032-1306265

14. Rothenberg S, Cowles R (2012) The effects of laparoscopic Nissen fundoplication on patients with severe gastroesophageal reflux disease and steroid-dependent asthma. J Pediatr Surg 47(6):11011104. https://doi.org/10.1016/j.jpedsurg.2012.03.013

15. Stanton M, Andrews J, Grant H (2010) Adhesive snall bowel obstruction following anto-reflux surgery in children-Comparison of 232 lapaorscopic and open fundoplications. Eur J Pediatr Surg 1:11-13. https://doi.org/10.1055/s-0029-1237382

16. Diaz DM, Gibbons TE, Heiss K, Wulkan ML, Ricketts RR, Gold BD (2005) Antireflux surgery outcomes in pediatric gastroesophageal reflux disease. Am J Gastroenterol 100(8):1844-1852. https ://doi.org/10.1111/j.1572-0241.2005.41763.x

17. Shah SR, Jegapragasan M, Fox MD, Prince JM, Segura BJ, Kane TD (2010) A review of laparoscopic Nissen fundoplication in children weighing less than $5 \mathrm{~kg}$. J Pediatr Surg 45(6):1165-1168. https://doi.org/10.1016/j.jpedsurg.2010.02.078

18. Thatch KA, Yoo EY, Arthur LG III, Finck C, Katz D, Moront M, Prasad R, Vinocur C, Schwartz MZ (2010) A comparison of laparoscopic and open Nissen fundoplication and gastrostomy placement in the neonatal intensive care unit population. J Pediatr Surg 45(2):346-349. https://doi.org/10.1016/j.jpedsurg.2009.10.073

19. Barsness KA, St Peter SD, Holcomb GW III, Ostlie DJ, Kane TD (2009) Laparoscopic fundoplication after previous open abdominal operations in infants and children. J Laparoendosc Adv Surg Tech A 19(Suppl 1):S47-S49. https://doi.org/10.1089/ lap.2008.0131.supp

20. Kane TD, Brown MF, Chen MK, Members of the ANTC (2009) Position paper on laparoscopic antireflux operations in infants and children for gastroesophageal reflux disease. American Pediatric Surgery Association. J Pediatr Surg 44(5):1034-1040. https://doi. org/10.1016/j.jpedsurg.2009.01.050

21. Papandria D, Goldstein SD, Salazar JH, Cox JT, McIltrot K, Stewart FD, Arnold M, Abdullah F, Colombani P (2015) A randomized trial of laparoscopic versus open Nissen fundoplication in children under two years of age. J Pediatr Surg 50(2):267-271. https://doi. org/10.1016/j.jpedsurg.2014.11.014

22. Shariff F, Kiely E, Curry J, Drake D, Pierro A, McHoney M (2010) Outcome after laparoscopic fundoplication in children under 1 year. J Laparoendosc Adv Surg Tech A 20(7):661-664. https://doi.org/10.1089/lap.2010.0213

23. Rothenberg SS (2013) Two decades of experience with laparoscopic nissen fundoplication in infants and children: a critical evaluation of indications, technique, and results. J Laparoendosc Adv Surg Tech A 23(9):791-794. https://doi.org/10.1089/ lap.2013.0299

24. Laje P, Blinman TA, Nance ML, Peranteau WH (2017) Laparoscopic fundoplication in neonates and young infants: failure rate and need for redo at a high-volume center. J Pediatr Surg 52(2):257-259. https://doi.org/10.1016/j.jpedsurg.2016.11.019 